\section{TRANSCRIPTOMIC CHANGES IN CANCER PATIENTS TREATED WITH IMMUNE-CHECKPOINT INHIBITORS}

${ }^{1}$ Dmitrii Shek*, ${ }^{2}$ Bo Gao, ${ }^{3}$ Joey Lai, ${ }^{2}$ Won-Hee Yoon, ${ }^{4}$ Tania Moujaber, ${ }^{4}$ Adnan Nagrial, ${ }^{5}$ Matteo Carlino, ${ }^{3}$ Scott Read, ${ }^{1}$ Golo Ahlenstiel. 'Western Sydney University, Sydney, New South Wales, Australia; ${ }^{2}$ Blacktown Hospital, Blacktown, Australia; ${ }^{3}$ Westmead Institute for Medical Research, Sydney, Australia; ${ }^{4}$ Westmead Hospital, Sydney, Australia; ${ }^{5}$ The University of Sydney, Sydney, Australia

Background Immune-checkpoint inhibitors (ICIs) are monoclonal antibodies that block inhibitory CTLA-4/PD-1 signalling pathways and thus boost cytotoxic $\mathrm{T}$ cell antitumor activity. ICIs have been proven effective in various malignancies, but there is a lack of knowledge regarding factors associated with ICI efficacy and safety. This study aims to examine transcriptomic changes in cancer patients treated with ICIs and their potential association with related clinical outcomes.

Methods This is a prospective multicentre cohort study (NCT04631731) recruiting cancer patients treated with (1) ICI monotherapy; (2) ICI dual therapy; (3) ICI + kinase inhibitor; (4) ICI + platinum-doublet chemotherapy. Peripheral blood is collected at baseline and 6-8 weeks after first ICI treatment as well as after the development of immune-related adverse events (irAEs, grade 2 and higher). Whole transcriptome sequencing (Novaseq S4 300 cycle lane, Illumina) was performed and followed by functional annotation using the ConsensusPath-DB platform.

Results 22 patients were recruited to the study and had paired blood taken. Two patients had developed grade 3-4 irAEs. RNA sequencing analysis identified 3,000 genes that were significantly dysregulated at week 6-8 after ICI commencement as compared to pre-treatment in $n=20$ recruited patients without irAEs (figure 1). Functional annotation established that 132 pathways were associated with the identified set of dysregulated genes. Among them: (1) pre-NOTCH processing in Golgi, (2) Interleukin-15 signalling; (3) STAT5 activation, and (4) RORA activation of gene expression possessed a gene set enrichment of at least $80 \%$ and $\mathrm{p}<0.01$. In 2 patients with grade 3 immune-mediated hepatitis, both treated with combination of CTLA-4/PD-1 inhibitors, analysis revealed that 360 and 325 were 2 -fold up- and downregulated respectively upon onset of toxicity as compared to both pre-treatment and 1week post-steroid treatment. Interestingly, this gene set possessed minimal overlap when compared to genes dysregulated in patients without irAEs. Moreover, functional annotation established different pathways that were associated with toxicity. The highest enrichment scores belonged to pathways regulating cell cycle and apoptotic pathways driven by CDC25A, p53 and BCL-2, among others.

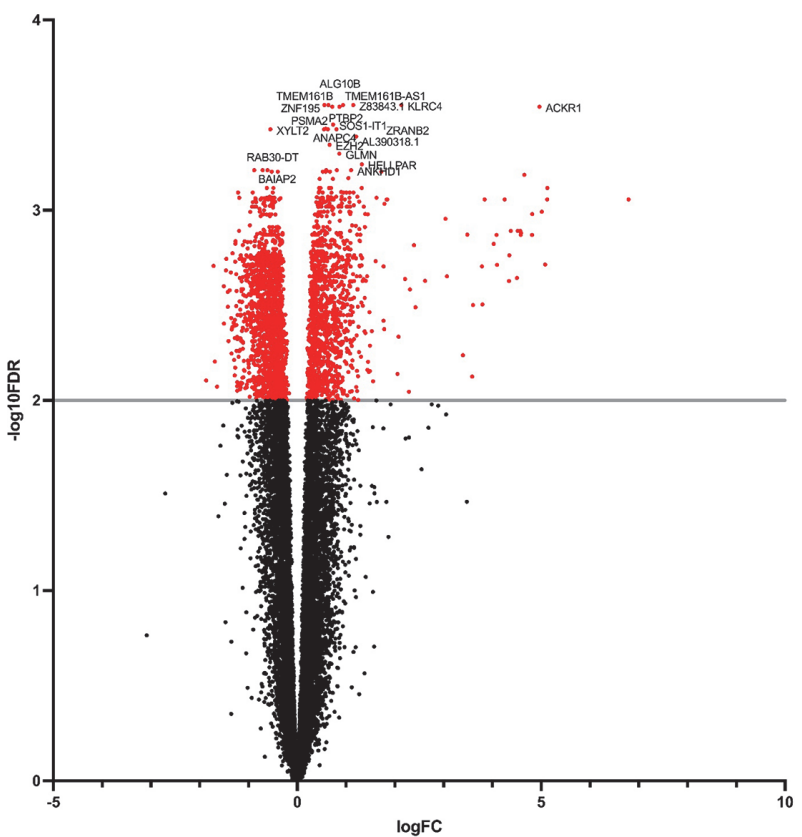

Abstract 522 Figure 1 Volcano plot representing the differentially expressed genes

The figure representing differentially expressed genes elucidated in this pilot study. $\mathrm{N}=3000$ genes were significantly dysregulated between pre- and week 6-8 post-IO commencement.

Conclusions The preliminary analysis of the first 22 patients recruited to NCT04631731 confirms that ICI treatment interferes with expression of coding and non-coding RNAs. Importantly, patients with and without irAEs show different patterns of transcriptomic changes as well as variability among activated cellular pathways. This data emphasises the need for further exploration and validation of transcriptomic changes in a larger cohort. In the near future, RNA signatures may be utilised as biomarkers to rapidly and accurately diagnose irAEs.

Acknowledgements N/A

Trial Registration ClinicalTrials.Gov identification number: NCT04631731

\section{REFERENCES}

$\mathrm{N} / \mathrm{A}$

Ethics Approval This study has been approved by the Western Sydney Local Health District (WSLHD) Human Research Ethics Committee on the November 9th, 2020 to be conducted at Blacktown and Westmead Public Hospitals of the WSLHD, Sydney, NSW, Australia.

Consent Each participant recruited to this translational study has provided written consent approved on the November 6th, 2020 (MASTER version) by the WSLHD HREC.

http://dx.doi.org/10.1136/jitc-2021-SITC2021.522 